66

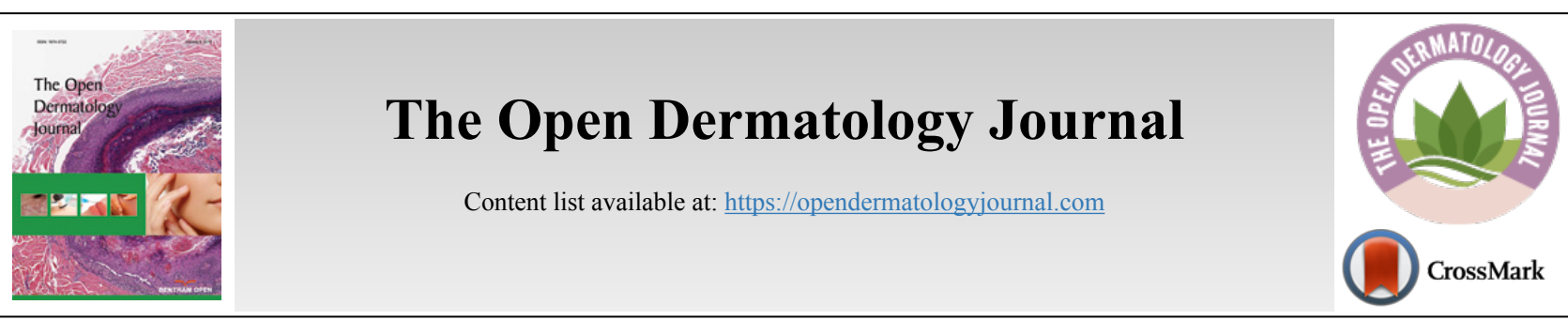

REVIEW ARTICLE

\title{
Burn Care in the Era of Rapid Enzymatic Debridement: Challenging the Dogma that Healing Beyond 21 Days Results in Hypertrophic Scarring
}

\author{
Lior Rosenberg ${ }^{1,2, *, \#, ~ Y a r o n ~ S h o h a m ~}{ }^{3,4, \#}$, Stan Monstrey ${ }^{5}$, Henk Hoeksema ${ }^{5}$, Jeremy Goverman ${ }^{6}$, William Hickerson ${ }^{7}$, \\ Ilaria Mataro ${ }^{8}$ and Adam J. Singer ${ }^{9}$ \\ ${ }^{l}$ Cleft Lip \& Palate \& Craniofacial Deformities Unit, Department of Plastic Surgery, Meir Hospital, Kfar Saba, Israel \\ ${ }^{2}$ Medical Department, MediWound LTD, Yavne, Israel \\ ${ }^{3}$ Department of Plastic Surgery and Burn Unit, Soroka University Medical Center, Beer Sheba, Israel \\ ${ }^{4}$ Faculty of Health Sciences, Ben-Gurion University of the Negev, Beer Sheba, Israel \\ ${ }^{5}$ Department of Plastic Surgery and Burn Unit, Ghent University Hospital, Ghent, Belgium \\ ${ }^{6}$ Sumner Redstone Burn Center, Department of Surgery, Massachusetts General Hospital, Harvard Medical School, Boston, MA, USA \\ ${ }^{7}$ Department of Plastic Surgery, College of Medicine, University of Tennessee Health Science Center, Department of Medicine, Firefighters Regional \\ Burn Center, Regional One Health, Memphis, TN, USA \\ ${ }^{8}$ Department of Plastic Surgery and Burn Unit, Antonio Cardarelli Hospital, Naples, Italy \\ ${ }^{9}$ Department of Emergency Medicine, Stony Brook University, Stony Brook, NY, USA
}

\begin{abstract}
:
Deep burns are characterized by the presence of a necrotic eschar that delays healing and results in a local and systemic inflammatory response and following healing by secondary intention: heavy scarring. Early surgical debridement followed by grafting was a major advance in deep burn care and is now the standard of care, reducing mortality and hypertrophic scarring. Eschars have alternatively been managed by non-surgical, autolytic debridement, which often results in infection-inflammation, slow epithelialization, granulation tissue formation and subsequent scarring. Studies based on these traditional approaches have demonstrated an association between delayed wound closure (beyond 21 days) and scarring. Early enzymatic debridement with NexoBrid (NXB) followed by appropriate wound care is a novel minimally invasive modality that challenges the well-accepted dictum of a high risk of hypertrophic scarring associated with wound closure that extends beyond 21 days. This is not surprising since early and selective removal of only the necrotic eschar often leaves enough viable dermis and skin appendages to allow healing by epithelialization over the dermis. In the absence of necrotic tissue, healing is similar to epithelialization of clean dermal wounds (like many donor sites) and not healing by the secondary intention that is based on granulation tissue formation and subsequent scarring. If and when granulation islands start to appear on the epithelializing dermis, they and the inflammatory response generally can be controlled by short courses (1-3 days) of topically applied low strength corticosteroid ointments minimizing the risk of hypertrophic scarring, albeit with wound closure delayed beyond the magic number of 21 days. Results from multiple studies and field experience confirm that while deep burns managed with early enzymatic debridement often require more than 21 days to reepithelialize, long-term cosmetic results are at least as good as with excision and grafting.
\end{abstract}

Keywords: Burns, Minimal invasive modality for burn care, Time to wound closure, Scarring, Enzymatic débridement, Debridase, Debriding gel dressing, NexoBrid, DGD, Enzymatic escharotomy, Enzymatic surgery.

\begin{tabular}{|l|l|l|l} 
Article History & Received: May 4, 2021 & Revised: September 15, 2021 & Accepted: October 12, 2021
\end{tabular}

\section{INTRODUCTION}

The aim of burn care is to achieve the best functional and aesthetic outcome with minimal complications and costs to both patients and the healthcare system. Burn management requires a fundamental understanding of wound biology followed by an appropriate therapeutic response to the profound local and systemic processes following thermal injury.

* Address correspondence to this author at the Department of Plastic Surgery, Cleft Lip \& Palate \& Craniofacial Deformities Unite, Meir Hospital Kfar Saba, Kfar Saba, Israel; Tel: + (972) 54-521-2269; Fax: +(972) 779714111;

E-mail: liorr@mediwound.com

"Both the authors contributed equally.
The thermally coagulated tissue (termed the burn eschar) rapidly induces a cascade of local and systemic pathological changes. Many of the treatment strategies employed during the long healing period aim to minimize complications secondary to the burn eschar. Following the primary trauma, the intense local inflammatory response starts, with the burn eschar inducing additional inflammation and systemic insults, extending the damage to the surrounding, non-injured tissues. This may result in the transformation and death of the zones of hyperemia and stasis surrounding the central zone of original necrosis, known as "burn wound propagation" "progression" or 
"conversion". The eschar generally becomes heavily contaminated within two to three days, forming a source for local and systemic inflammation and infection, often leading to a systemic inflammatory response syndrome (SIRS), bacteremia and sepsis. The eschar, as well as bacteria, elicit a strong inflammatory response from the surrounding viable tissue attracting neutrophils, which release proteolytic enzymes and inflammatory mediators. This results in maceration of the eschar, causing it to slough or separate from the newly formed underlying granulation tissue in a process referred to as autolysis. This process is slow and exposes patients to a number of eschar-related complications, including wound infection, sepsis and death. The amount of inflammation elicited by the eschar depends on its overall size and thickness as well as the amount of time that the eschar remains as a substrate for bacterial activity. The prolonged inflammatoryinfectious process induces the formation of granulation and later on, hyper-granulation tissue that often evolves into dense, deforming and hypertrophic scars (HS). Delaying the removal of the eschar increases the incidence and severity of these eschar related complications, especially in more extensive burns [1 - 6].

Since the eschar cannot stretch under the rapidly forming tissue edema (a component of the inflammatory response) there is an abrupt increase in the interstitial/compartment pressure. The mounting pressure compromises the venous return increasing congestion, swelling and pressure, further compromising perfusion. The reduction in perfusion extends the initial cutaneous burn damage, further reducing the local blood supply to vital structures, such as nerves and muscles, causing a burn-induced compartment syndrome (BICS). Early (within hours) diagnosis of the developing BICS and release of the constricting eschar by performing a surgical escharotomy may prevent and resolve this process; however, delay or hesitation in the diagnosis of BICS and reluctance to perform an adequate surgical escharotomy (especially in borderline cases and where experienced burn surgeons are not readily available) may lead to irreversible damage. Surgical escharotomy reduces the compartment pressure but has the potential to harm important underlying structures (i.e., nerves, vessels), leading to additional complications, permanent disabilities and scars [7 - 9].

Burn depth and extent is not static but is a dynamic process where injury progression depends on factors, such as the severity of the initial thermal insult, anatomic site, time from injury, intensity of the inflammatory and infectious processes, vasoconstriction and use of vasoconstricting agents, skin resilience and looseness, as well as physical factors, such as local skin tension, pressure, desiccation and cold, which may dramatically extend the original damage [10]. Estimation of burn size, depth and BICS is essential for a diagnosis-based treatment strategy, but in most cases, this cannot be accurately performed early after injury until the dense opaque eschar that covers the wound bed has been removed and the secondary damage has completed its course.

To prevent eschar-related complications and to initiate the healing process, the entire eschar needs to be removed as early as possible. Eschar removal (escharectomy, otherwise known as debridement) is the first stage of any wound care process. "Immediate" (within hours post-injury) eschar removal may improve the likelihood of survival of the zone of stasis, and reverse the zone of hyperemia. It also may attenuate, or avoid many related local and systemic inflammatory reactions as well as prevent or release BICS [11 - 14]. "Early" eschar removal (between the first and third day) will not prevent/release BICS early enough and may not provide all of the immunological and inflammatory modulation benefits; however, it still provides infection source-control, reduces the bacterial load and may prevent critical colonization, reducing inflammation/infection and their outcomes. Delaying eschar removal up to the seventh day after injury exposes the patient to eschar-related complications, but if combined with autografting, it may still prevent healing by secondary intention and the resulting scarring, ultimately improving the long-term results. Thus, the decision is not whether or not to remove the eschar, but when and how.

Prior to the era of early excision, non-surgical debridement and "eschar separation" (sloughing, autolysis) were practiced. As early as 1943, a specialist advisory committee of the Surgeon General of the US Army and Navy, under the chairmanship of A.O. Whipple, recognized the need to develop a means for early surgical excision as well as the need for "chemical" methods for "hastening the separation of the irreversibly damaged skin" [15]. This was followed by intense investigation of potential debriding enzymes and chemicals, but at present, only collagenase has remained in the market [15].

In 1970, tangential excision followed by autografting was "officially" introduced by Zora Janzekovic [16]. This method has become the current standard of care (SOC) for deep burns: surgical tangential excision followed by split thickness skin grafting (STSG). Surgical excision is carried down into the healthy intact tissue in order to ensure that no trace of the eschar remains. It has been estimated that up to $30-50 \%$ of the excised tissue is healthy and still viable (mainly dermis) [17]. This viable tissue could be essential for spontaneous wound healing by re-epithelialization over the dermis. In order to preserve some of this dermis, more selective surgical procedures (dermabrasion, hydro-surgery) can be used. These are slow, operator-dependent procedures that may save some of the otherwise sacrificed dermis but require general anesthesia and result in loss of blood and heat. The surgically debrided raw wound bed, which has little or no dermis, requires immediate coverage in order to avoid desiccation and secondary tissue necrosis and permanent epidermal as well as dermal replacement. This is achieved by autografting with a STSG, which provides permanent epidermal and some dermal coverage (although without dermal appendages) that closes the wound by primary intention. This still results in up to an $80 \%$ incidence of HS at the graft's edges and within its' meshed interstices. Scarring of the donor site is also a potential complication of this approach.

Thus, burn surgical procedures are long and traumatic, leading to loss of non-injured surrounding tissues, blood and heat. In addition, the need for pre-operative fasting further compromises patient nutrition and healing. The need for 
surgery also requires challenging anesthesia and recovery, as well as highly skilled surgical personnel, sophisticated facilities and abundant resources. In an attempt to minimize operative complications, an unstable patient may end up with fascial excisions and grafting, ultimately leading to an even more morbid operation with even more healthy tissue removed. While it is true that the STSG donor site is "only" a partial thickness skin defect that usually heals spontaneously within 2 weeks and with relatively minimal scarring, the sheer size of these donor sites and healing process is associated with considerable morbidity (mainly pain and sometimes scarring) not always fully-appreciated by physicians.

In an effort to avoid this high cost of burn surgery, especially in burns of uncertain or indeterminant depth or in unstable patients, a "conservative", non-surgical, autolytic approach, is often selected to initiate the treatment until the final surgical or non-surgical burn care strategy is chosen. This "wait and see" approach is a slow, gradual process of eschar decomposition where some areas may still be covered by the decomposing eschar while other areas, already devoid of eschar, are granulating and epithelializing. This process usually leads to both local and systemic complications. The gradual development of hypergranulation tissue often results in HS, especially if left to heal spontaneously by secondary intention. Although granulation tissue can very well support autografting, it still may develop into contracted scar tissue even under the graft itself [18].

\section{ASSOCIATION OF TIME TO WOUND CLOSURE AND SCARRING WITH THE TRADITIONAL SURGICAL AND NON-SURGICAL APPROACHES}

Striving to identify which healed burns require compression therapy, Deitch et al. retrospectively studied a cohort of 100 patients. They found that non-debrided burns treated conservatively requiring more than 21 days to heal spontaneously developed HS in $78 \%$ of the cases. In contrast, burn wounds that closed between 14 and 21 days developed HS in only $33 \%$ of the cases [19]. Thus, Deitch concluded that a time to wound closure greater than 21 days was responsible for HS.

This chronological threshold became the current dogma that a time to wound closure (TWC) greater than 21 days generally results in HS $[1-5,20]$. Therefore, burns that are deemed to require more than 21 days to heal spontaneously (large deep partial or full thickness) are typically managed by surgical debridement followed by STSG. Since early clinical determination of burn depth is often inaccurate, laser Doppler imaging (LDI) [21 - 24] was developed to help predict which burns would heal spontaneously in less than 21 days. LDI detects the rich capillary blood flow of the superficial papillary dermis that is the hallmark of a superficial burn, which results in healing within less than 21 days and minimal HS. However, even superficial partial thickness burns may have a thin overlying eschar which, if not removed, may sometimes result in inflammation, infection and subsequently, hypergranulation and HS, albeit less extensive, than with thicker eschars and deeper burns.

Of note, all previous studies have focused on burns in which the eschar remained for variable periods of time, either with the conservative or the surgical approach. This is in distinct contrast to other surgical wounds, such as STSG donor sites or deep abrasions, in which there is no eschar but only exposed dermis of varying thicknesses. These wounds usually heal spontaneously and rarely result in HS unless they become infected or desiccate. The TWC in these wounds is directly related to the depth of injury and the amount of remaining dermis (with its epithelial appendages), as well as the treatment strategy.

\section{THE ASSOCIATION BETWEEN TIME TO WOUND CLOSURE AND SCARRING AFTER EARLY ENZYMATIC DEBRIDEMENT WITH NEXOBRID}

Recently, a new bromelain-based enzymatic debridement agent: NexoBrid (NXB, Yavneh, Israel), has become available in some parts of the world. NXB is an effective, fast-acting and selective mixture of proteolytic enzymes [25 - 28], which is applied topically to the entire burn wound (regardless of depth and eschar thickness) as early as possible (practically on admission), dissolving eschars of all thicknesses. NXB consists of a concentrate of proteolytic enzymes enriched in Bromelain. The mechanism of action is via the specificity of the different proteolytic enzymes to denatured tissue, thus being able to selectively debride the different non-viable components within 4 hours, without harming viable tissue within this timeframe. In most cases, debridement is completed after a single 4-hour session within hours after injury and before inflammation and infection can ensue. Enzymatic debridement, being specific to the burn eschar, can be applied on all burns without a previous diagnosis of their depth, removing only the thermally injured eschar and sparing viable tissues, including the dermis. This three-dimensional specificity results in the preservation of varying amounts of dermis covering large areas of an escharfree wound bed. This differs from the traditional surgical debridement (tangential excision or dermabrasion) with its century old strategy and tools in which sequential, indiscriminate layers of tissue are removed until bleeding is seen. In wounds where the skin's entire thickness has been burned, enzymatic debridement exposes the subdermal tissues (i.e., fat, fascia or muscle), which requires permanent coverage with a skin graft, dermal substitutes or flaps. Wounds with a sufficient quantity of remaining viable dermis, can be treated like STSG donor sites, aiming toward spontaneous epithelialization (originating from surviving skin adnexa and the wound edges) over the dermis with little and often no scar formation.

Three general principles should be kept in mind when treating the exposed dermal bed: (1). Protecting it from desiccation, (2). Offering the neo-epidermis adequate conditions to propagate (a viable surface, adequate coverage, moisture and mechanical protection) and; (3). Controlling any granulation tissue as soon as it appears [25 - 28]. Since spontaneous epithelialization may take longer than graft-take, often, after $\sim 2$ weeks, small islands of granulation may appear all over the wound bed. This granulation tissue, if treated for 2-3 days with topical low potency preparations of corticosteroid ointment, will retreat, allowing epithelialization to progress and the wound to close. Uncontrolled, this 
granulation may become hypertrophic, less susceptible to steroids and develop into scars. Of note, in the studies supporting the above principles, all the investigators employed pressure garments and silicone after wound closure regardless of their closure strategy.

This approach, which includes very early selective enzymatic debridement, preservation of the non-injured dermis that is treated towards spontaneous re-epithelialization, controlling granulation tissue, and autografting of only full thickness defects and non-healing wounds, has been termed the Minimally Invasive Modality (MIM) of burn care (ISBI Jerusalem meeting 1998) [29]. Already then, we noticed that these wounds developed thinner and less exuberant granulation tissue and the final outcome of wound healing was much better than expected considering the burn depth and longer TWC.

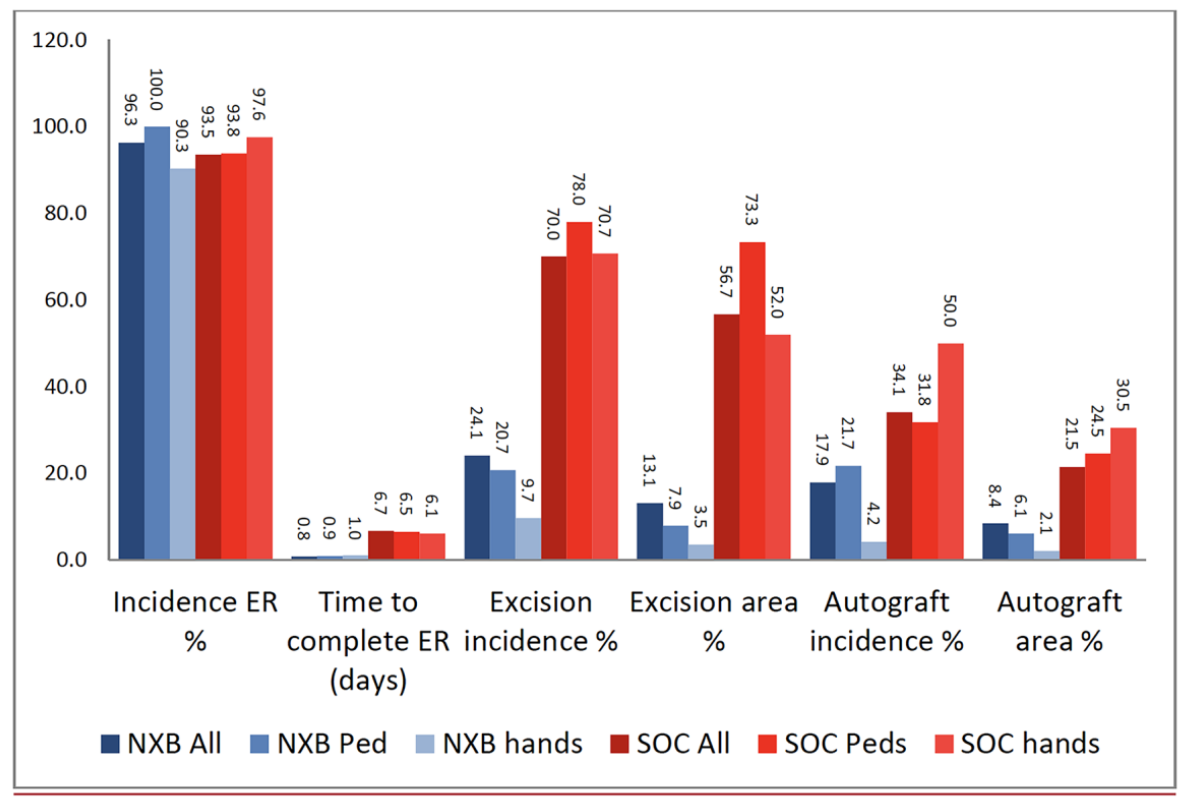

Fig. (1). Results of the phase $3 \mathrm{RCT}$, NexoBrid $v s$. SOC (surgical and non-surgical) for all wounds, pediatric patients, and hand burns $\mathrm{ER}=$ eschar removal.

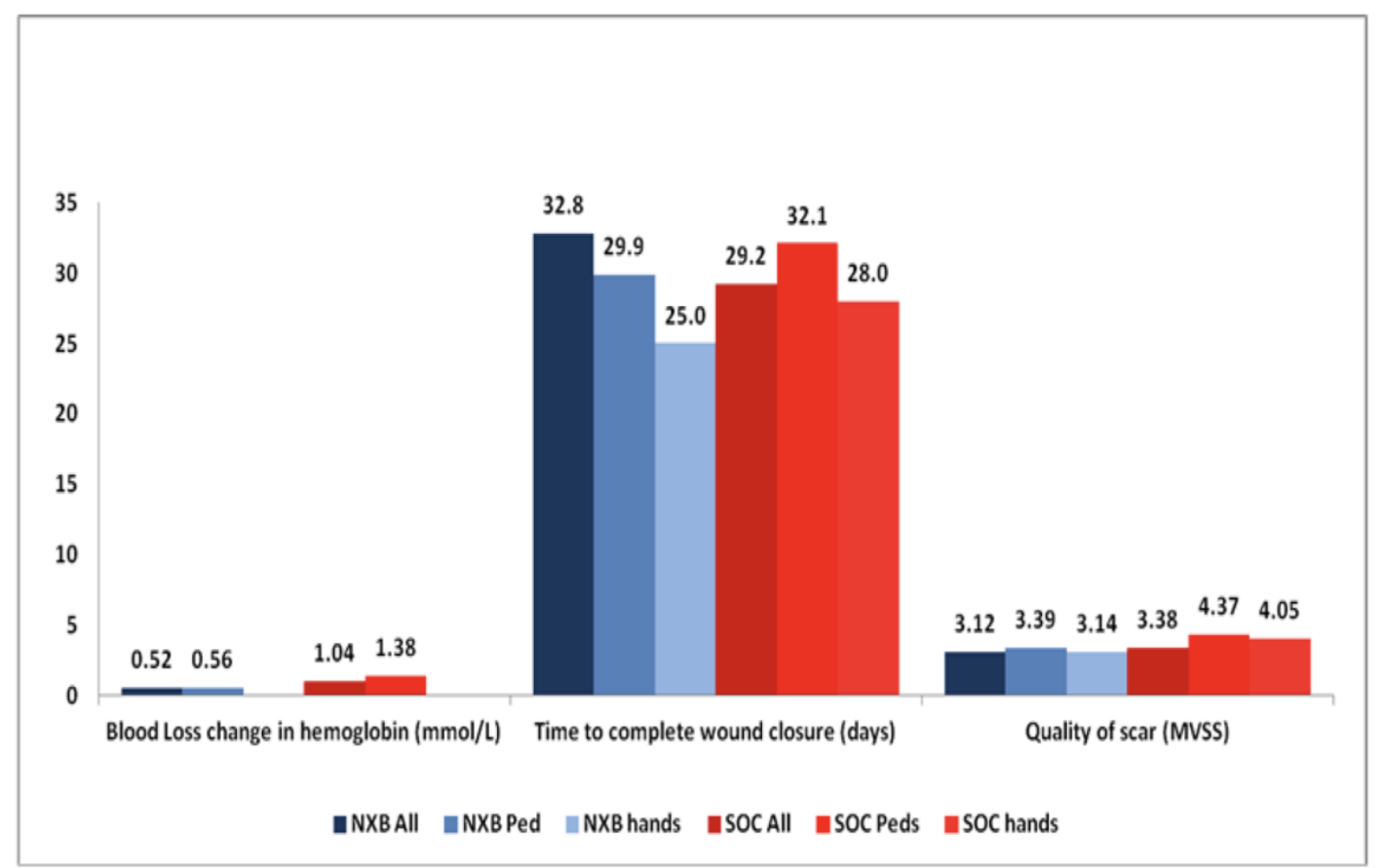

Fig. (2). Additional results of the phase $3 \mathrm{RCT}$, NexoBrid vs. SOC (surgical and non-surgical) for all wounds, pediatric patients, and hand burns. 
Over the last several years, a number of studies have compared the efficacy, safety and selectivity of early enzymatic debridement and the standard surgical approach both in animals [30 - 33] as well as in humans [26]. These results are summarized in a recent publication of seven studies, which were part of the development plan for NXB [34]. The results of the phase 3 RCT are presented in Figs. (1 and 2). The seven studies included 543 patients (including 110 children), of which 372 were treated with enzymatic debridement and 171 with the standard of care $[25,27,35]$. While none of the NXB treated circumferential extremity burns required escharotomy, $\sim 10 \%$ of the burns in the SOC arm underwent escharotomy.
The findings of all these studies are further supported by multiple post-marketing clinical studies and reports, including a European Consensus Paper, confirming the earlier results [28, 36 - 45].

Hoeksema et al. [46] described their cohort of 37 separate burn wounds that were assessed by LDI as deep and in need of excision and grafting. However, following early and selective enzymatic debridement, these burns were found to have enough intact dermis to allow spontaneous epithelialization after a mean of 27.6 days (range of 16-57 days) with only 4 (10.8\%) wounds developing some HS [44] (Figs. 3 and 4).
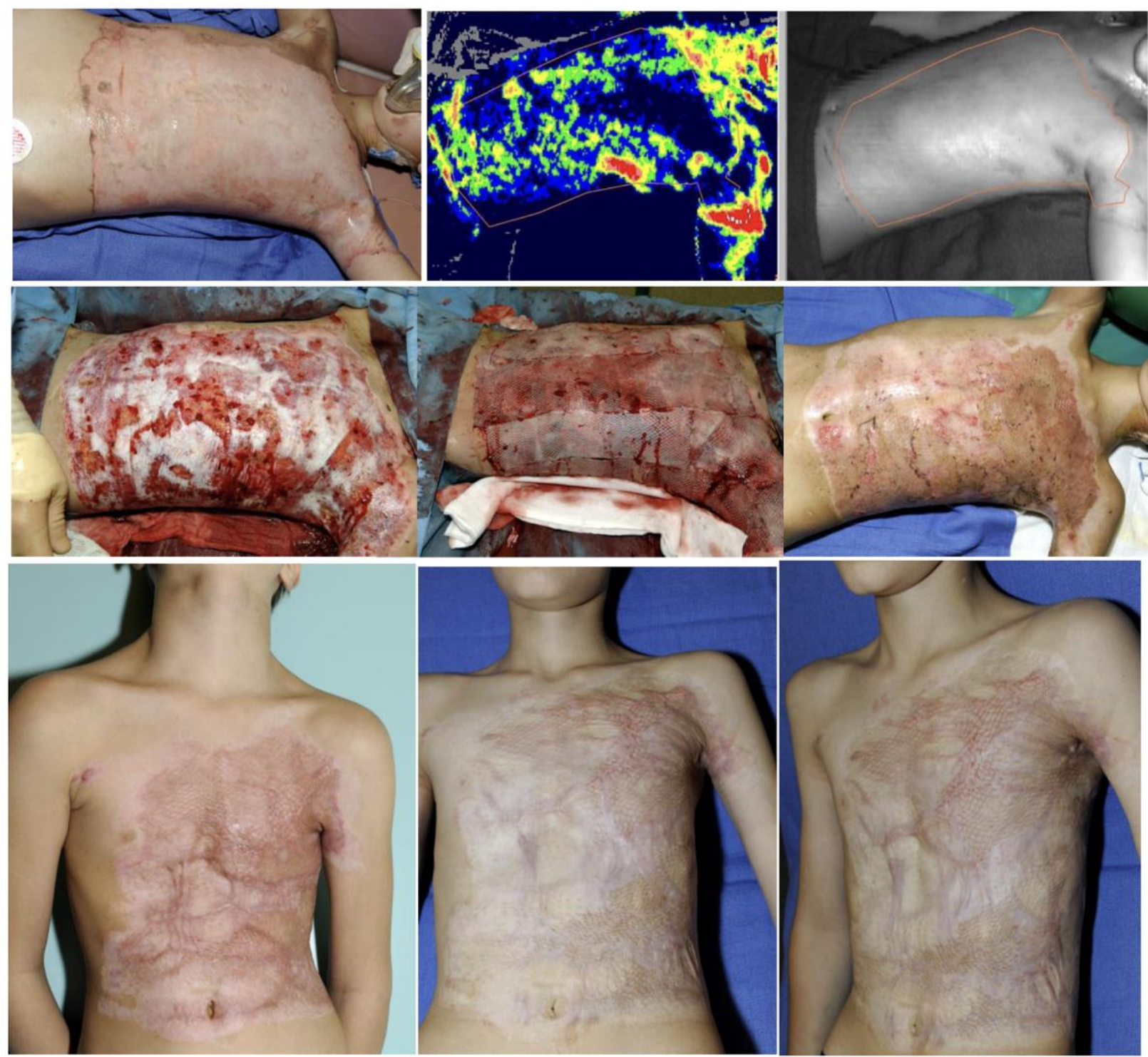

Fig. (3). Representative patient treated with the SOC burn care.

(a). Day 1 post injury (PI). (b). Day 2 PI: LDI image demonstrating burn areas predicted not to heal in 21 days without hypertrophic scaring thus, requiring excision and autografting. (c). Day 9 PI: tangential excision (d). mesh 1/1.5 autografting. (e). Day 45 PI: >95\% wound closure. (f). Month 3 PI: follow up visit. (g). One-year PI. The donor site pictures are not available. Courtesy of Drs. Stan Monstrey and Henk Hoeksema from Gent (Belgium) burn center. 

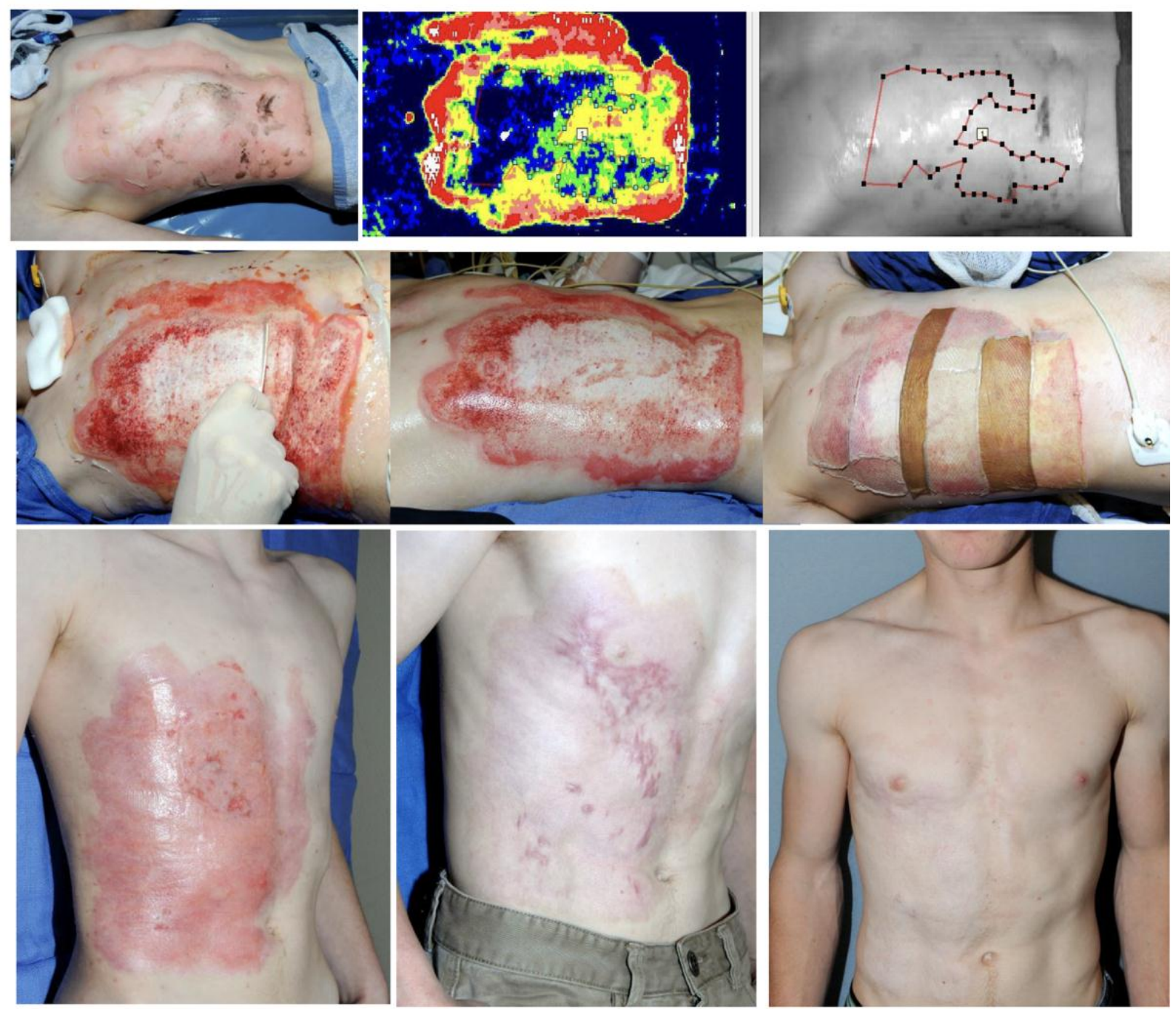

Fig. (4). Representative patient treated with NexoBrid.

(a). Admission on day of injury. (b). Day 2 post injury (PI): LDI image demonstrating areas prone to delayed ( $>21$ days) healing and hypertrophic scaring. (c). Day 3 PI: NexoBrid debridement 4 hours after its application: the dissolved eschar is wiped away using a wooden tongue depressor. (d). After NexoBrid debridement and saline soaking. (e). The wounds were covered with meshed allografts (two different donor sites) and treated conservatively thereafter (with fatty ointments and short, 2-3 day courses of corticosteroid ointment as soon as granulation tissue islands appeared). (f). Day 43 PI: $>95 \%$ wound closure by epithelialization over preserved dermis. (g). Three months PI: follow up visit showing scarring in some of the late-healing areas. (h). One-year PI: follow up visit.

Courtesy of Drs. Stan Monstrey and Henk Hoeksema from Gent (Belgium) burn center.

In the two burn patients presented Figs. (3 and 4), the etiology (flame), age, depth, size and site are similar, however treatment strategies are different: surgical SOC (TE \& meshed STSG) vs. NXB debridement and epithelialization. Wound closure is quite similar in both patients with $>95 \%$ wound closure by meshed/sheet autograft over the excised wound bed Fig. (3e and 3f) and meshed allografts (from two donors) over the NXB-debrided bed Fig. (4f and 4g). At three months scars appear around and within the grafts that distort them Fig. 3g) and at the last areas to epithelialize as immature, vascularized scars Fig. (4g). At one year each case matured in a different way: the scars around and insides the grafts are softer and whiter but still quite visible distorting the grafted area in an only too familiar appearance Fig. 3h). The scars of the NXB patient matured also but blended TE, tangential excision; STSG, splitthickness skin graftingNXB, NexoBrid. with the surrounding epithelialized dermis Fig. 4h). It seems that the quality of healing is related mainly to the dermis that is involved in this process.

The most significant and consistent findings of the above studies were that enzymatic debridement reduced the time to wound debridement, reduced the need for and the total area of surgical excision and autografting, reduced the incidence and area of donor sites, and prevented the need for escharotomy in deep circumferential extremity burns. In all studies, long-term cosmetic and functional outcomes after enzymatic debridement were at least as good as with the standard of care, even when wound closure was delayed. The results of these studies confirm the following:

(1) Clinical assessment of burn depth is often inaccurate: there is often more viable dermis than expected under the fresh 
eschar, even in the so-called "deep" burns.

(2) The standard surgical strategy of early excisional debridement of deep burns effectively removes the eschar and may prevent eschar-related complications. However, surgical debridement is traumatic and non-specific, often sacrificing much (if not all) of the viable dermis. As a result, surgically excised burns generally require autografting (or other permanent coverage).

(3) Early, fast, and selective enzymatic debridement can complete eschar removal as early as the day of admission ( $v s$. $\sim 1$ week in the SOC treated patients), preventing eschar related complications and allowing accurate visual diagnosis of the remaining exposed wound bed (Figs. 3 and 4).

(4) The surgical SOC may shorten the TWC, but the incidence of scarring, its area and quality (measure by the modified Vancouver scar scale) were not reduced (Figs. 1-3).

(5) Early and selective enzymatic debridement often results in very large areas of exposed, viable dermis in burns that were destined for surgical debridement and autografting. However, after enzymatic debridement, these wounds often can heal by spontaneous epithelialization, obviating the need for autografting with final results that are at least as good as (if not better than) excision and grafting despite a TWC longer than 21 days (Figs. 2-4).

(6) Granulation tissue can and should be controlled/modulated as soon as it appears by short (2-3 days) courses of a topical corticosteroid ointment. Hypergranulation should not be allowed and if granulation tissue can't be controlled, it should be excised and grafted as soon as possible.

(7) Early enzymatic debridement of circumferential burns of the extremities, which are prone to develop BICS, may prevent or resolve BICS.

(8) Many burns diagnosed as "deep" by LDI (in which excision and grafting were indicated based on the current standard of care) when enzymatically debrided, were found to have enough viable dermis to allow spontaneous epithelialization resulting in a good final outcome.

\section{DISCUSSION}

Healing of burns is a multifactorial, dynamic and complex process. The presence of a necrotic eschar, besides being a source of local and systemic complications, delays healing and increases the risk of granulation tissue formation followed by HS. The delay in healing is thought to be the etiology of excessive scarring. However, scarring is the result of a process based on an intense inflammatory response, often with superimposed infection, which is induced by the eschar and not by the delayed TWC. When the necrotic eschar is removed very early after injury, the eschar-related complications (including inflammation) can be mitigated. When enough viable dermis is present, keratinocytes from the epidermal appendages and surrounding uninjured edges, under the influence of numerous wound healing mediators and given adequate conditions (surface, protection and moisture), proliferate and migrate to re-establish the epidermal surface. Obviously, the time required to close (epithelialize) the debrided wound depends on the wound size, amount of remaining dermis and epithelial foci, as well as other host and wound factors, including the dynamics of the epithelialization process. Excisional debridement can in theory, allow very early eschar removal. However, very often the time to make this decision, prepare the patient, and organize the facilities stretches into many days. In addition to being resourcedependent, surgical excision or debridement is traumatic, resulting in blood and heat loss, and removal of viable dermis. The development of an effective, fast-acting and selective enzymatic agent (NXB) introduced a novel therapeutic option leading to a new clinical paradigm of very early (sometimes hours post injury) debridement where large areas of preserved dermis can heal by spontaneous epithelialization. We found that these wounds epithelialized well, apparently with reduced granulation tissue formation, in contrast to the healing of nondebrided wounds, which heal by secondary intention after eschar autolysis and the development of excessive granulation tissue. These wounds may close slower than the excised and autografted wounds, often requiring longer than 21 days to heal, but ultimately lead to good results, resembling other superficial to intermediate dermal wounds, such as donor sites (Figs. 3 and 4). However, it is important to recognize that these wounds can start to granulate, which can, and must be adequately modulated.

Table 1. Time to wound closure and hypertrophic scarring: conservative and surgical treatment (from Cubison et al.).

\begin{tabular}{|c|c|c|c|c|}
\hline $\begin{array}{c}\text { Days to } \\
\text { TWC }\end{array}$ & $\begin{array}{c}\text { \% HS (No. of } \\
\text { patients) } \\
\text { Conservative } \\
\text { management }\end{array}$ & $\begin{array}{c}\text { \% HS (No. of } \\
\text { patients) } \\
\text { TE\&STSG }\end{array}$ & $\begin{array}{c}\text { Difference } \\
\text { in \% HTS }\end{array}$ & $\begin{array}{c}\% \text { HS (No. } \\
\text { of } \\
\text { patients) } \\
\text { All } \\
\text { wounds }\end{array}$ \\
\hline$<10$ & $0(4)$ & $0(1)$ & & $0(5)$ \\
\hline $10-14$ & $2(8)$ & $33(15)$ & +31 & $8(39)$ \\
\hline $15-21$ & $20(20)$ & $19(15)$ & -1 & $20(35)$ \\
\hline $22-25$ & $28(14)$ & $54(11)$ & +26 & $40(25)$ \\
\hline $26-30$ & $75(8)$ & $64(11)$ & -11 & $68(19)$ \\
\hline$>30>30$ & $94(47)$ & $88(32)$ & -6 & $92(69)$ \\
\hline
\end{tabular}

TWC, time to wound closure; HS, hypertrophic scarring; TE\&STSG, tangential excision and split thickness skin grafting.

One of the more frequently-quoted papers on the issue of TWC and scarring is by Deitch et al. [19]. In 1983, they attempted to identify burn variables that predicted the need for compression therapy. They found an association between TWC of conservatively treated burns and HS. However, in their paper, they also referred to previous studies that mentioned the relationship between burn depth and the potential for HS, hypothesizing that by limiting the period of the inflammatory response (by excising granulation tissue) fibroplasia and scarring decreased too [47, 48]. Though Deitch and other authors felt that burn depth may be a predictor of scarring, the diagnosis of burn depth was not perceived reliable enough. In addition, burns did not behave as expected: a third of the "superficial" burns scarred heavily and a third of the "deep" burns did not scar at all [20]. Additional factors possibly associated with HS included younger age, dark skin color, and local factors, such as wound tension and infection. Deitch et al. published their retrospective series of 100 patients (245 
separate burn wounds) with a wide range of ages, races, genders and anatomical sites. All burns were treated conservatively from admission to wound closure with twice daily application of silver sulfadiazine (SSD). The chest, upper extremities and feet were more prone to HS as well as wounds that took longer than 21 days to heal. However, no relationship was found between age and skin color and the need for compression therapy. Thus, he chose a very clear criterion, a TWC greater than 21 days, as the most important predictor of HS and an indicator for compression therapy. All the cited authors in his publication recognized the inaccuracy of clinical burn depth assessment and the heterogeneity of all the factors involved, which contribute to the formation of scars. The source of the HS, i.e., the eschar related inflammatory/infectious autolytic processes that are directly related to granulation tissue formation and subsequent scarring, were not discussed. However, as early as the 1970 s, Linares et al. [49] followed by Krizek and Robson [50] openly blamed granulation tissue for HS. Unfortunately, the focus in the literature has been exclusively on the easier and clearer target: TWC, which we now know to be only one factor among several, contributing to scarring. Four years later, Deitch's team published a prospective study on the variables associated with post burn HS, considering TWC as the most important factor [51]. They argued that burns limited to the papillary dermis would not scar and that deeper burns involving the reticular dermis were prone to hypertrophic scarring, ignoring the role of the eschar itself as well as other previous publications [20].

Others have also recognized that the relationship between TWC and scarring is complex and involves numerous other factors [52]. In 2006, Cubison et al. studied a cohort of 337 children with scalds treated conservatively or surgically in which the scars were monitored for up to 5 years. The study correlated wound depth (or eschar thickness) and HS (Table 1) and concluded that there is a low risk of HS in scalds that heal in less than 21 days. However, since surgically treated burns (which were closed within 21 days) were also associated with HS, the authors concluded that surgery should be reserved for scalds likely to take longer than 21 days to heal. In this study, there was a direct relationship between TWC and frequency of HS (from no scars in wounds closing within 10 days, to almost all $(92 \%)$ cases in wounds requiring more than a month to close). In the group of patients managed surgically, there also was a gradual increase in scarring over time. Interestingly, in burns that healed within 10-14 days, scarring was more frequent in the surgical than the non-surgical group (33 vs. $2 \%$ ). In burns that healed within 22-25 days, scarring was still more frequent in the surgical group (54 vs. $28 \%$, respectively). Even for burns that healed most slowly ( $>30$ days), the difference in scarring (somewhat worse in the conservative arm) was not great (88vs. 94\%, Table 1) [52].

In the two similar cases presented in Figs. (3 and $\mathbf{4}$ ) (one patient treated with the surgical SOC and the other with NXB enzymatic debridement and epithelialization over salvaged dermis) one can appreciate that grafting does not prevent scarring, though in practice, with $100 \%$ graft take, such wounds may be considered "closed" after a few days.
Measuring TWC of burns is also not simple as the healing process is not homogeneous throughout the entire wound surface. The "completely" healed wound continues to undergo modulation and maturation that may include sprouting of cutaneous adnexae through the healed surface with temporary minute "openings". Thus, while initially appearing closed, the wound is not $100 \%$ closed. Wounds in this stage are clinically/medically closed and not prone to infection, which may happen in the very early phases of wound healing, when necrotic tissue is present. The healed burn wounds are often treated with a variety of protective, humidifying, or scar modulating dressings for months. The FDA defines "complete wound closure" as: "skin re-epithelialization without drainage or dressing requirements confirmed at two consecutive study visits, two weeks apart" [53] and does not consider these phenomena and the need for long-term dressings of healed burns [54]. The American Burn Association consensus statement that was created to redefine burn related measures and was based on the vast clinical experience of its 88 senior authors defines wound closure as "1. Time (in days) to complete (>95\%) wound closure; and 2. Size (TBSA) of open wounds at the time of hospital discharge" [54]. Considering these different definitions for wound closure, the fact that patients may be discharged prior to wound closure, the challenge of assessing TWC and that the time of assessment can be delayed by days or even weeks (depending on the follow-up regimen), the accuracy of TWC (in days) is questionable. Tredget et al. [55] elegantly reviewed the formidable intricate lattice of factors that influence each other and the ever-changing process of wound healing and scarring. Besides factors such as age, gender, genetic make-up, and wound location, they point out (again) the importance of inflammation: "prolonged inflammation of slowly healing burn wounds seems to be the most contributing factor". Numerous local and systemic factors, cytokines and growth factors together with a variety of cells play important roles. This includes three different fibrocyte lines (in the skin adnexae, superficial dermis and deep dermis) [56, 57], each seemingly with different biological behaviors. This dynamic "inflammatory" process is triggered by the thermal trauma itself, but not less important are the roles of toll-like receptors (TLR). The TLRs not only activate the immune system by sensing the presence of damage associated molecular pattern (DAMPs) ligands released from the damaged, necrotic tissue, but also switch the response from normal wound healing to fibrosis in many different organs and tissues. At present, 10 different TLRs have been identified that bind specific ligands, such as TLR2 that recognizes gram-positive bacteria and TLR4 that senses gram-negative bacteria by binding lipopolysaccharides (LPS). TLR4, stimulated by LPS, was found to induce extracellular matrix deposition and liver fibrosis. Recently, cutaneous hypertrophic scar fibroblasts have also been found to have increased expression of TLR4 mRNA and surface receptors $[58,59]$. Thus, it seems that the escharrelated impact of the DMAP-TLR pathway and the inflammatory processes are more profound and far-reaching on the molecular and cellular levels than just the days to wound closure.

Commonly studied DAMPs include high-mobility group 
box 1 (HMGB1), a nuclear protein that is released by dead or dying cells after burn injury. The release of HMGB1 leads to activation of the innate immune system via the recognition of DAMPs by pathogen recognition receptors (PRRs). Consequently, a host of cytokines are produced, leading to inflammation and a proinflammatory state. Following burn injury, HMGB1 may favor the TLR2 and TLR4 pathways. Another DAMP associated with burn injury is the cytosol S100 protein known to interact with TLR4 and Advanced Glycosylation End product-specific Receptor (AGER) following burn injury [60]. Activation of these damageassociated molecular pathways also drives fibrosis in a variety of diseases, such as systemic sclerosis [61] and in other organs beyond the skin, such as the kidneys [62] and heart [63].

The differences between the spontaneous healing process and outcomes of burns (that comprise burn eschar) and graft donor sites of the same depth may corroborate the DAMP activated TLR hypothesis. Mid-dermal burns heal but often with scars, while similar depth donor sites epithelialize with less scarring. The depth of injury may be similar, but the presence of the eschar and eschar related inflammatory/infectious complications of the autolytic process induce different healing patterns.

The importance of the systemic influences of the eschar is exemplified in reports of immediate (or primary) eschar removal, where total eschar removal reduced morbidity and mortality by reducing inflammation and later, infection $[11,14$, 64]. Recently Hassan et al. [65] openly challenged the dogma of the direct relationship between TWC and scarring, reporting that $85 \%$ of the wounds that took longer than 21 days to heal (some as long as 88 days) did not develop HS. He points out that all wounds that developed HS had clinical signs of infection.

These and other reports, which have led to the concept that delayed wound closure is the main cause for HS, appear oversimplified and even misleading. The increased TWC of nondebrided wounds is the result of extremely complex healing and inflammatory processes and not the cause of these processes. As a result, in order to influence scarring, we should modulate the factors and processes that are the cause of HS and not the time to healing that is their result. Thus, the choice between the two strategies: epithelialization of uncontrolled eschar-related inflamed wounds or surgical excision and grafting of wounds (with resultant scars around and within the grafts as well as at the donor sites) seems to be unsatisfactory. The minimally invasive burn care modality characterized by early selective debridement that exploits the healing potential while modulating inflammation and infection is neither novel nor specific to NXB. In 1984, Holmes noted that burns thought to be deep and in need of excision and grafting can be dermabraded down to the remnant mixed dermis/full thickness bed after a two-week period of conservative care, allowing the eschar to soften. This bed could then be grafted or treated conservatively, aiming for epithelialization from the edges and few remaining skin adnexa [62, 63]. When islands of granulation appeared, they were dermabraded again and again until complete epithelialization or autografting closed the wound. In this study, the rather late debridement did not prevent the inflammatory- infectious process and the formation of granulation tissue, with HS and contractures reported in $37 \%$ and $4 \%$ of wounds respectively, in spite of generous grafting ( $78.5 \%$ of the cases). Of note: TWC was always under 21 days after abrasion ( 11 days for abraded, 9 days for abraded and grafted and 12 days for abraded and mesh grafted wounds). Despite the delay in debridement, Holmes' papers [66, 67] shed light on some of the main issues associated with enzymatic debridement-based minimally invasive modality: The diagnosis of mixed burns, the loss of precious dermis by tangential excision, the potential for a clean viable dermal bed to epithelialize and the need to control recurrent foci of granulation tissue (by dermabrasion in his report) are challenging. The enzymatic debridement-based minimally invasive modality differs from Holmes' approach by the very early enzymatic debridement of all wounds regardless of their depth. This non-surgical, selective eschar removal (that probably reduces burn propagation), prevents or reduces the eschar-related inflammation and infection and preserves more dermis that is allowed to epithelialize. Unfortunately, we cannot compare Holmes' results of delayed debridement with the very early selective debridement approach used in our series. Holmes' also supports the concept of surgically controlling granulation tissue, whereas we suggest using topical corticosteroid ointment to modulate inflammation and granulation tissue formation. We found it very effective, especially if the steroid treatment started very early, when only a few islands of granulation tissue were present (generally around days 12-14). Short, 2-3-day courses of topical steroids will reduce these islands allowing epithelialization to progress. If new islands appear, another short course of topical steroids controls them too, promoting epithelialization even further (as recently described by one of the centers using NXB [68]. Wound care should not allow the transformation of these minute, thin granulation islands into hypergranulation tissue with the consequent scarring [69 - 71] Bromelain (contained within NXB) is known to have strong anti-inflammatory properties [70, 71]. However, it is not clear how important this feature is in reducing scarring compared to the early removal of the eschar and the use of topical steroids to control the granulation process of the NXB-debrided wounds.

The two most basic therapeutic principles in medicine are primum non nocere (first do no harm) and restitutio ad integrum (restoration to original condition). From the patient's perspective, the first concern is to not worsen their condition by avoiding unnecessary and potentially destructive surgery and its associated pain and complications. The second concern is to achieve an optimal scar that limits disfigurement and dysfunction. The famous saying that "epidermis is life and dermis is quality of life" is the basis for life-saving burn surgery and excisional debridement with dermal losses and extensive use of STSG. Since the 70s, this strategy has saved many lives but left many with skin graft and donor site-related scars. As elaborated earlier, the final outcome of scar quality depends on multiple tissue and physical factors (not all elucidated yet) such as the presence of dead tissue, viable dermis, regenerative factors, inflammation/infection, moisture, local wound tension and pressure as well as the genetic predisposition of the injured patient. While using autografts, 
we should not forget the scarring that occurs at the transition between grafts and normal skin as well as in the incisions within the grafts due to meshing or Meek expansion. Thus, TWC per-se, is probably one of the less important factors contributing to scarring. TWC seems to be less relevant to patients as long as the best possible final outcome is reached. The additional few days required until wound closure seems to be even less relevant to patients considering that most are discharged before their wounds are closed. The hospital length of stay itself depends on many factors, such as available and used dressings, reimbursement strategy, nature and availability of outpatient facilities, patient related factors as well as TWC. The eventual return to the pre-injury state depends even less on TWC as convalescence, physical and psychological therapy, scar maturation, scar modulation and final scar quality are long processes (months to years), which continue long after wounds are closed. Thus, the additional few days or weeks required till wound closure have little long-term impact. The ability to return to work may be positively or negatively influenced by TWC. However, other medical, social, psychological and economic factors may be just as important, whereas skin grafting (considering the time for complete healing of the grafted areas and the donor site) does not always hasten the ability to return to work. TWC is easier to assess (though as previously discussed, this is neither easy nor accurate) than the various tissue and inflammation factors, cellular lines and the long-term, final outcomes (scarring, function, quality of life, etc.), which are months to years ahead. Thus, TWC is used as an endpoint in various wound healing studies, but its clinical relevance and benefit to patients (considering all these factors) could and should be questioned.

\section{CONCLUSION}

The association between delayed (greater than 21 days) wound closure and HS is based on the traditional non-surgical debridement approach, where the necrotic eschar is not removed and macerates (autolysis), resulting in a vicious cycle of inflammation-infection-granulation leading to late wound closure and resulting in HS. Only the more superficial burns heal earlier than 21 days and with minimal scarring due to the minimal necrotic eschar and the related reduced infectioninflammation. While surgery (surgical debridement followed by STSG) saves lives by removing the noxious eschar and may close the wound earlier, preventing the scars associated with healing by secondary intention, it does not eliminate scarring and HS. With the early selective enzymatic debridement based minimally invasive modality, the eschar is removed very early after injury preventing the eschar related complications, especially the vicious cycle of inflammation-infection, leaving the non-injured viable dermis intact and allowing spontaneous epithelialization over it as seen in the healing of donor sites. This may require more than 21 days, but with minimal inflammation and modulation of granulation tissue formation, HS can usually be avoided or minimized. Other than survival, the single most important measure of optimal burn care is the outcome of healing. The quality of healing (i.e., scarring) and, in this paper, its relationship to the arbitrary or at least oversimplified threshold of 21 days to wound closure is challenged. We propose that the final quality of healing as well as the biological and pathological factors contributing to it, should be determined and used as measures for clinical practices and study endpoints.

\section{CONSENT FOR PUBLICATION}

Not applicable.

\section{FUNDING}

None.

\section{CONFLICT OF INTEREST}

LR and YS have financial interests in MediWound where they serve as a chief medical officer (LR) and a consultant (YS). None of the other investigators and authors had any financial interest in NXB or MediWound (Yavneh, Israel) that sponsored studies 2-7.

\section{ACKNOWLEDGEMENETS}

Declared none.

\section{REFERENCES}

[1] Lewis GM, Heimbach DM. Evaluation of the burn wound: management decision. Total burn care 4th. ed., ed. D.N. Herndon 2012.

[2] Sheridan RL. Comprehensive treatment of burns. Curr Probl Surg 2001; 38(9): 657-756.

[http://dx.doi.org/10.1016/S0011-3840(01)70019-6]

[PMID: 11568825

[3] White paper surgical management of the burn wound and use of skin substitutes. American Burn Association 2009.

[4] Orgill DP. Excision and skin grafting of thermal burns. N Engl J Med 2009; 360(9): 893-901.

[http://dx.doi.org/10.1056/NEJMct0804451] [PMID: 19246361]

[5] Silver GM, Klein MB, Herndon DN, et al. Standard operating procedures for the clinical management of patients enrolled in a prospective study of inflammation and the host response to thermal injury. J Burn Care Res 2007; 28(2): 222-30.

[http://dx.doi.org/10.1097/BCR.0B013E318031AA44]

[PMID: 17351437]

[6] Tredget EE. Management of the acutely burned upper extremity. Hand Clin 2000; 16(2): 187-203.

[http://dx.doi.org/10.1016/S0749-0712(21)00196-7] [PMID: 10791166]

[7] Orgill DP, Piccolo N. Escharotomy and decompressive therapies in burns. J Burn Care Res 2009; 30(5): 759-68.

[http://dx.doi.org/10.1097/BCR.0b013e3181b47cd3] [PMID: 19692906]

[8] Wong L, Spence RJ. Escharotomy and fasciotomy of the burned upper extremity. Hand Clin 2000; 16(2): 165-174, vii. [vii.]. [http://dx.doi.org/10.1016/S0749-0712(21)00194-3] 10791164]

[9] Saffle JR, Zeluff GR, Warden GD. Intramuscular pressure in the burned arm: Measurement and response to escharotomy. Am J Surg 1980; 140(6): 825-31.

[http://dx.doi.org/10.1016/0002-9610(80)90126-9] [PMID: 7457708]

[10] Heimbach D, Engrav L, Grube B, Marvin J. Burn depth: A review. World J Surg 1992; 16(1): 10-5.

[http://dx.doi.org/10.1007/BF02067108] [PMID: 1290249]

[11] Voinchet V, Bardot J, Echinard C, Aubert JP, Magalon G. Advantages of early burn excision and grafting in the treatment of burn injuries of the anterior cervical region. Burns 1995; 21(2): 143-6.

[http://dx.doi.org/10.1016/0305-4179(95)92141-X] [PMID: 7766325]

[12] Barret JP, Wolf SE, Desai MH, Herndon DN. Total burn wound excision of massive paediatric burns in the first 24 hours post-injury. Ann Burn Fire Disas 1999; 12: 25-7.

[13] Barret JP, Herndon DN. Effects of burn wound excision on bacterial colonization and invasion. Plast Reconstruct Surg 2003; 111(2): 744-50.

[14] Barret JP, Herndon DN. Modulation of inflammatory and catabolic 
responses in severely burned children by early burn wound excision in the first 24 hours. Arch Surg 2003; 138(2): 127-32.

[http://dx.doi.org/10.1001/archsurg.138.2.127] [PMID: 12578404]

[15] Klasen HJ. A review on the nonoperative removal of necrotic tissue from burn wounds. Burns 2000; 26(3): 207-22.

[http://dx.doi.org/10.1016/S0305-4179(99)00117-5] [PMID: 10741585]

[16] Janzekovic Z. A new concept in the early excision and immediate grafting of burns. J Trauma 1970; 10(12): 1103-8.

[http://dx.doi.org/10.1097/00005373-197012000-00001] [PMID: 4921723]

[17] Gurfinkel R, Rosenberg L, Cohen S, et al. Histological assessment of tangentially excised burn eschars. Can J Plast Surg 2010; 18(3): e33-6. [http://dx.doi.org/10.1177/229255031001800303] [PMID: 21886431]

[18] McDonald WS, Deitch EA. Hypertrophic skin grafts in burned patients: A prospective analysis of variables. J Trauma 1987; 27(2): $147-50$.

[http://dx.doi.org/10.1097/00005373-198702000-00008] [PMID: 3546710]

[19] Deitch EA, Wheelahan TM, Rose MP, Clothier JI, Cotter JO. Hypertrophic burn scars: Analysis of variables. J Trauma 1983; 23(10): 895-.

[20] Page RE, Barclay TL. Correlation of scald depth and hypertrophic scar formation. Burns 1980; 7: 173-5. [http://dx.doi.org/10.1016/0305-4179(81)90060-7]

[21] Rosenberg L, Molcho J, Dotan Y, Baruchin A, Mahler D. Use of the Doppler effect in visible laser light to assess tissue viability by capillary blood flow. Ann Plast Surg 1982; 8(3): 206-12.

[http://dx.doi.org/10.1097/00000637-198203000-00007] [PMID: 7103370]

[22] Burke-Smith A, Collier J, Jones I. A comparison of non-invasive imaging modalities: Infrared thermography, spectrophotometric intracutaneous analysis and laser Doppler imaging for the assessment of adult burns. Burns 2015; 41(8): 1695-707.

[http://dx.doi.org/10.1016/j.burns.2015.06.023] [PMID: 26421694]

[23] Jaskille AD, Shupp JW, Jordan MH, Jeng JC. Critical review of burn depth assessment techniques: Part I. Historical review. J Burn Care Res 2009; 30(6): 937-47.

[http://dx.doi.org/10.1097/BCR.0b013e3181c07f21] [PMID: 19898102]

[24] Jaskille AD, Ramella-Roman JC, Shupp JW, Jordan MH, Jeng JC. Critical review of burn depth assessment techniques: Part II. Review of laser doppler technology. J Burn Care Res 2010; 31(1): 151-7. [http://dx.doi.org/10.1097/BCR.0b013e3181c7ed60] [PMID: 20061851]

[25] Rosenberg L, Lapid O, Bogdanov-Berezovsky A, et al. Safety and efficacy of a proteolytic enzyme for enzymatic burn debridement: A preliminary report. Burns 2004; 30(8): 843-50. [http://dx.doi.org/10.1016/j.burns.2004.04.010] [PMID: 15555800]

[26] Rosenberg L, Krieger Y, Silberstein E, et al. Selectivity of a bromelain based enzymatic debridement agent: A porcine study. Burns 2012; 38(7): 1035-40.

[http://dx.doi.org/10.1016/j.burns.2012.02.011] [PMID: 22385643]

[27] Rosenberg L, Krieger Y, Bogdanov-Berezovski A, Silberstein E, Shoham Y, Singer AJ. A novel rapid and selective enzymatic debridement agent for burn wound management: A multi-center RCT. Burns 2014; 40(3): 466-74.

[http://dx.doi.org/10.1016/j.burns.2013.08.013] [PMID: 24074719]

[28] Hirche C, Citterio A, Hoeksema H, et al. Eschar removal by bromelain based enzymatic debridement $\left(\right.$ Nexobrid $\left.^{\mathbb{B}}\right)$ in burns: An European consensus. Burns 2017; 43(8): 1640-53.

[http://dx.doi.org/10.1016/j.burns.2017.07.025] [PMID: 29033046]

[29] Rosenberg L. A debridase based minimal invasive modality (MIM) or the Primum Non Nocere (PNN) Protocol for burn treatment: A preliminary report. In: The 10th International Congress of the ISBI. Jerusalem, Israel. 1998.

[30] Krieger Y, Rosenberg L, Lapid O, et al. Escharotomy using an enzymatic debridement agent for treating experimental burn-induced compartment syndrome in an animal model. J Trauma 2005; 58(6): 1259-64.

[http://dx.doi.org/10.1097/01.TA.0000169867.08607.F1] [PMID: 15995479]

[31] Singer AJ, Taira BR, Anderson R, McClain SA, Rosenberg L. The effects of rapid enzymatic debridement of deep partial-thickness burns with Debrase on wound reepithelialization in swine. J Burn Care Res 2010; 31(5): 795-802.

[http://dx.doi.org/10.1097/BCR.0b013e3181eed48e] [PMID:
20661148

[32] Singer AJ, McClain SA, Taira BR, Rooney J, Steinhauff N, Rosenberg L. Rapid and selective enzymatic debridement of porcine comb burns with bromelain-derived Debrase: Acute-phase preservation of noninjured tissue and zone of stasis. J Burn Care Res 2010; 31(2): 304-9.

[http://dx.doi.org/10.1097/BCR.0b013e3181d0f4d4] [PMID: 20182376]

[33] Singer AJ, Taira BR, Anderson R, McClain SA, Rosenberg L. Reepithelialization of mid-dermal porcine burns after rapid enzymatic debridement with Debrase ${ }^{\circledR}$. J Burn Care Res 2011; 32(6): 647-53. [http://dx.doi.org/10.1097/BCR.0b013e31822dc467] [PMID: 21979853]

[34] Rosenberg L, Shoham Y, Krieger Y, et al. Minimally invasive burn care: A review of seven clinical studies of rapid and selective debridement using a bromelain-based debriding enzyme (Nexobrid ${ }^{\circledR}$ ). Ann Burns Fire Disasters 2015; 28(4): 264-74. [PMID: 27777547]

[35] Krieger Y, Bogdanov-Berezovsky A, Gurfinkel R, Silberstein E, Sagi A, Rosenberg L. Efficacy of enzymatic debridement of deeply burned hands. Burns 2012; 38(1): 108-12.

[http://dx.doi.org/10.1016/j.burns.2011.06.002] [PMID: 22103988]

[36] Koller J, Bukovcan P, Orság M, Kvalténi R, Gräffinger I. Enzymatic necrolysis of acute deep burns--report of preliminary results with 22 patients. Acta Chir Plast 2008; 50(4): 109-14.

[PMID: 19408486]

[37] Cordts T, Horter J, Vogelpohl J, Kremer T, Kneser U, Hernekamp JF. Enzymatic debridement for the treatment of severely burned upper extremities - Early single center experiences. BMC Dermatol 2016; 16(1): 8 .

[http://dx.doi.org/10.1186/s12895-016-0045-2] [PMID: 27342276]

[38] Cherubino M, Pellegatta I, Sallam D, Pulerà E, Valdatta L. Enzymatic debridement after mobile phone explosion: A case report. Ann Burns Fire Disasters 2016; 29(4): 273-5.

[PMID: 28289361]

[39] Schulz A, Perbix W, Shoham Y, et al. Our initial learning curve in the enzymatic debridement of severely burned hands-Management and pit falls of initial treatments and our development of a post debridement wound treatment algorithm. Burns 2017; 43(2): 326-36.

[http://dx.doi.org/10.1016/j.burns.2016.08.009] [PMID: 28341257]

[40] Palao R, Aguilera-Sáez J, Serracanta J, Collado JM, Dos Santos BP, Barret JP. Use of selective enzymatic debridement agent (Nexobrid) for wound management: Learning curve. World J Dermatology 2017; 6(2): 32-41.

[http://dx.doi.org/10.5314/wjd.v6.i2.32]

[41] Schulz A, Shoham Y, Rosenberg L. Enzymatic versus traditional surgical debridement of severely burned hands: A comparison of selectivity, efficacy, healing time, and three-month scar quality. J Burn Care Res 2017; 38(4): e745-55.

[http://dx.doi.org/10.1097/BCR.0000000000000478]

[42] Rigueros Springford L, Creasy H, Cubison T, Dheansa B. A novel technique of NexoBrid ${ }^{\mathrm{TM}}$ application to burns on the hands. Burns 2017; 43(5): 1132-3

[http://dx.doi.org/10.1016/j.burns.2017.02.013] [PMID: 28330824]

[43] Schulz A, Fuchs PC, Rothermundt I, et al. Enzymatic debridement of deeply burned faces: Healing and early scarring based on tissue preservation compared to traditional surgical debridement. Burns 2017; 43(6): 1233-43.

[http://dx.doi.org/10.1016/j.burns.2017.02.016] [PMID: 28363663]

[44] Giudice G, Filoni A, Maggio G, Bonamonte D, Vestita M. Cost analysis of a novel enzymatic debriding agent for management of burn wounds. BioMed Research International 2017; 5.

[http://dx.doi.org/10.1155/2017/9567498]

[45] Chesebro JH, Fuster V, Elveback LR, et al. Effect of dipyridamole and aspirin on late vein-graft patency after coronary bypass operations. $\mathrm{N}$ Engl J Med 1984; 310(4): 209-14.

[http://dx.doi.org/10.1056/NEJM198401263100401] [PMID: 6361561]

[46] Hoeksema H. Challenging the 21 Day Golden Rule for Wound Closure: Lessons Learned from Rapid Enzymatic Eschar Removal in Deep Burns Assessed by LDI, in ABA annual meeting presentation. Available from: https://ir.mediwound.com/node/7221

[47] Koonin AJ. The aetiology of keloids: A review of the literature and a new hypothesis. S Afr Med J 1964; 38: 913-6. [PMID: 14225677]

[48] Ketchum LD. Hypertrophic scars and keloids. Clin Plast Surg 1977; 4(2): 301-10. 
[http://dx.doi.org/10.1016/S0094-1298(20)30584-8] [PMID: 856531]

[49] Linares HA, Kischer CW, Dobrkovsky M, Larson DL. On the origin of the hypertrophic scar. J Trauma Acute Care Surg 1973; 13(1): 70-5.

[50] Krizek TJ, Robson MC. Foreword. Clin Plast Surg 1979; 6(4): 491-2.

[51] McDonald WS, Deitch EA. Hypertrophic skin grafts in burned patients: A prospective analysis of variables. J Trauma 1987; 27(2): 147-50.

[http://dx.doi.org/10.1097/00005373-198702000-00008] [PMID: 3546710]

[52] Cubison TCS, Pape SA, Parkhouse N. Evidence for the link between healing time and the development of hypertrophic scars (HTS) in paediatric burns due to scald injury. Burns 2006; 32(8): 992-9. [http://dx.doi.org/10.1016/j.burns.2006.02.007] [PMID: 16901651]

[53] Guidance for industry chronic cutaneous ulcer and burn wounds developing products for treatment Available from: https://www.fda.gov/media/71278/download

[54] Gibran NS, Wiechman S, Meyer W, et al. Summary of the 2012 ABA Burn Quality Consensus conference. J Burn Care Res 2013; 34(4): 361-85.

[http://dx.doi.org/10.1097/BCR.0b013e31828cb249] [PMID: 23835626]

[55] Tredget EE, Levi B, Donelan MB. Biology and principles of scar management and burn reconstruction. Surg Clin North Am 2014; 94(4): 793-815.

[http://dx.doi.org/10.1016/j.suc.2014.05.005] [PMID: 25085089]

[56] Sorrell JM, Baber MA, Caplan AI. Site-matched papillary and reticular human dermal fibroblasts differ in their release of specific growth factors/cytokines and in their interaction with keratinocytes. J Cell Physiol 2004; 200(1): 134-45.

[http://dx.doi.org/10.1002/jcp.10474] [PMID: 15137066]

[57] Dunkin CSJ, Pleat JM, Gillespie PH, Tyler MPH, Roberts AHN, McGrouther DA. Scarring occurs at a critical depth of skin injury: Precise measurement in a graduated dermal scratch in human volunteers. Plast Reconstr Surg 2007; 119(6): 1722-32.

[http://dx.doi.org/10.1097/01.prs.0000258829.07399.f0] [PMID: 17440346]

[58] Piccinini A, Midwood K. DAMPening inflammation by modulating TLR signalling. Mediators Inflamm 2010.

[59] Wang J, Hori K, Ding J, et al. Toll-like receptors expressed by dermal fibroblasts contribute to hypertrophic scarring. J Cell Physiol 2011; 226(5): 1265-73

[http://dx.doi.org/10.1002/jcp.22454] [PMID: 20945369]

[60] Comish PB, Carlson D, Kang R, Tang D. Damage-associated molecular patterns and the systemic immune consequences of severe thermal injury. J Immunol 2020; 205(5): 1189-97. [http://dx.doi.org/10.4049/jimmunol.2000439] [PMID: 32839211]

[61] Bhattacharyya S, Varga J. Endogenous ligands of TLR4 promote unresolving tissue fibrosis: Implications for systemic sclerosis and its targeted therapy. Immunol Lett 2018; 195: 9-17.

[http://dx.doi.org/10.1016/j.imlet.2017.09.011] [PMID: 28964818]

[62] Anders HJ, Schaefer L. Beyond tissue injury-damage-associated molecular patterns, toll-like receptors, and inflammasomes also drive regeneration and fibrosis. J Am Soc Nephrol 2014; 25(7): 1387-400. [http://dx.doi.org/10.1681/ASN.2014010117] [PMID: 24762401]

[63] Turner NA. Inflammatory and fibrotic responses of cardiac fibroblasts to myocardial damage associated molecular patterns (DAMPs). J Mol Cell Cardiol 2016; 94: 189-200.

[http://dx.doi.org/10.1016/j.yjmcc.2015.11.002] [PMID: 26542796]

[64] Barret JP, Wolf SE, Desai MH, Herndon DN. Total burn wound excision of massive paediatric burns in the first 24 hours post-injury. Annal Burn Fire Disast 1999; 12: 25-7.

[65] Hassan S, Reynolds G, Clarkson J, Brooks P. Challenging the dogma: Relationship between time to healing and formation of hypertrophic scars after burn injuries. J Burn Care Res 2014; 35(2): e118-24. [http://dx.doi.org/10.1097/BCR.0b013e31829b330a]

[66] Holmes J, Muir I, Rayner C. A hypothesis of the healing of deep dermal burns and the significance for treatment. Br J Surg 70(10) 611-3.

[67] Holmes JD, Rayner CR. The technique of late dermabrasion for deep dermal burns. Implications for planning treatment. Burns 1984; 10(5): 349-54.

[http://dx.doi.org/10.1016/S0305-4179(84)80008-X]

[PMID: 6744081]

[68] Jaeger M, Harats M, Kornhaber R, Aviv U, Zerach A, Haik J. Treatment of hypergranulation tissue in burn wounds with topical steroid dressings: A case series. Int Med Case Rep J 2016; 9(9): 241-5. [PMID: 27570466]

[69] Roland P. The formation and management of middle ear granulation tissue in chronic ear disease. Ear Nose Throat J 83(1 Suppl): 5-8. [http://dx.doi.org/10.1177/014556130408301s02]

[70] Mandrea E. Topical diflorasone ointment for treatment of recalcitrant, excessive granulation tissue. Dermatol Surg 1998; 24(12): 1409-10. [http://dx.doi.org/10.1111/j.1524-4725.1998.tb00024.x] [PMID: 9865213]

[71] McShane DB, Bellet JS. Treatment of hypergranulation tissue with high potency topical corticosteroids in children. Pediatr Dermatol 2012; 29(5): 675-8.

[http://dx.doi.org/10.1111/j.1525-1470.2012.01724.x] [PMID: 22612258]

(C) 2021 Rosenberg et al.

This is an open access article distributed under the terms of the Creative Commons Attribution 4.0 International Public License (CC-BY 4.0), a copy of which is available at: https://creativecommons.org/licenses/by/4.0/legalcode. This license permits unrestricted use, distribution, and reproduction in any medium, provided the original author and source are credited. 